\title{
LOWER BOUNDS OF RATES OF DECAY FOR SOLUTIONS TO THE NAVIER-STOKES EQUATIONS
}

\author{
MARIA E. SCHONBEK
}

To Ron

\section{INTRODUCTION}

We study the asymptotic behavior of solutions to the Navier-Stokes equations in two and three space dimensions

$$
\begin{gathered}
u_{t}+u \nabla u+\nabla p=\Delta u, \\
\operatorname{div} u=0 .
\end{gathered}
$$

Our earlier work $[4,5]$ dealt with the upper bounds on the $L^{2}$ decay rates of solutions to the Navier-Stokes equations in three space dimensions with large data. It was established that if $u_{0} \in L^{2} \cap L^{p}, 1 \leq p<2$, then

$$
|u(\cdot, t)|_{L^{2}}^{2} \leq C(1+t)^{-\alpha(p)},
$$

where $\alpha(p)=\frac{3}{4}(2 / p-1)$ and the constant $C$ depends on the $L^{2}$ and $L^{p}$ norms of the initial data $u_{0}$.

This paper deals with the more subtle problem of deriving lower bounds on the energy decay rates. We show that for a certain class of initial data the solutions $u(x, t)$ to the 2D and 3D Navier-Stokes equations admit an algebraic lower bound on the energy decay. Specifically, there are two cases to consider. In the first case, the average of the initial data $\int u_{0} d x$ is nonzero. This case was treated in the earlier paper [5] where it was established that

$$
|u(\cdot, t)|_{L^{2}}^{2} \geq C(1+t)^{-n / 2}
$$

for $n=2,3$. In the second case the average is zero, i.e., the Fourier transform at the origin is zero. Here the lower bound on the velocity of decay rate depends on the order of the zero of the initial data. More precisely, if the zero is of order one and the data $u_{0} \in L^{1} \cap H^{1}$ and certain weighted $L^{p}$ spaces described below then there is a lower bound of the form

$$
|u(\cdot, t)|_{L^{2}}^{2} \geq C(1+t)^{-\alpha(n)},
$$

Received by the editors July 1, 1989 and, in revised form, July 24, 1990.

1991 Mathematics Subject Classification. Primary 35K55.

The work of the author was partially supported by National Science Foundation grants DMS8614887 and DMS 8610730. 
where $\alpha(n)=n / 2+1$ and $C$ depends on a few parameters of the data. If the zero is of order greater than one and the data is taken outside a set $M$ of radially equidistributed energy, then in two dimensions the lower bound will be described by (1.3). The lower bound obtained in 2D is uniform, the one in $3 \mathrm{D}$ is not. The estimate in two dimensions is sharp. An example suggested by A. Majda shows that there are solutions to the Navier-Stokes equations with data in $M$ that decay exponentially.

The algebraic lower bound is a consequence of the nonlinear structure of the equations. In contrast, solutions to the heat equation decay at an exponential rate if the initial data is highly oscillatory. The inertial term $\operatorname{div}(u \otimes u)$ in the Navier-Stokes equations appears to convert short waves into long waves, reducing the decay rate. Even for most cases of highly oscillatory initial data (i.e., containing just short waves), energy will be transmitted to the lower end of the scale, thereby producing long waves that reduce the decay rate of the solutions.

Our approach in the case of zero average data is first to find conditions for the data such that the corresponding solution to the heat equation decays at a very slow rate. These conditions will be met by the solution $u(x, t)$ of the Navier-Stokes at some time $t_{0} \geq 0$. That is, short waves are transformed into long waves. Hence the solution to the heat equation, which takes on as initial data $u\left(x, t_{0}\right)$ for some appropriate $t_{0} \geq 0$, has a lower bound on their rate of decay. Specifically, if $v$ is a solution of $v_{t}=\Delta v, v(x, 0)=u\left(x, t_{0}\right)$ then $|v(\cdot, t)|_{L^{2}}^{2} \geq(1+t)^{-\alpha(n)}$.

This information is used to insure that the solutions to the Navier-Stokes equations cannot decay any faster. The result follows using Fourier analysis of the Navier-Stokes equations. The argument relies on a technique that involves splitting the frequency space into two time-dependent sets. This technique was developed in [4] to study the upper bound.

For solutions in two spatial dimensions, the results are valid for classical solutions. For three dimensions, the results are valid for suitable Leray-Hopf solutions in the sense of Caffarelli, Kohn, and Nirenberg [1]. We expect that the results can be extended to $n$ dimensions, $n>3$, using the results of Wiegner [6], Kayikiya and Miyakawa [2].

\section{ESTIMATES ON SOLUTIONS TO THE HEAT EQUATIONS}

In this section we describe a class of initial data $D$, for which the solutions to the heat equations admit a lower bound on the $L^{2}$ decay rate. In $\S 3$, it is shown that if $u(x, t)$ is a solution to the Navier-Stokes in two spatial dimensions with data outside a set $M$ of radially equidistributed energy, then there is $t_{0} \geq 0$ for which $u\left(x, t_{0}\right) \in D$. Hence if $u\left(x, t_{0}\right)$ is taken as initial data, the corresponding solution to the heat equation will have a lower bound on the $L^{2}$ decay. This information will be used to obtain a lower bound on the $L^{2}$ rate of decay for solutions to the Navier-Stokes equations. For data in $M$, 
an example is given in $\S 5$ of a solution to the 2D Navier-Stokes equation that decays exponentially fast.

The section concludes with a lemma establishing an upper bound on the $L^{\infty}$ rate of decay for the gradient of the solutions to the heat equation for solutions that decay in $L^{2}$ at a given rate.

Theorem 2.1. Let $v_{0} \in L^{2}\left(\mathbf{R}^{n}\right)$. Let $v$ be a solution to the heat equation with data $v_{0}$. Suppose that there exist functions $l$ and $h$ such that the Fourier transform of $v_{0}$ for $|\xi| \leq \delta, \delta>0$, admits the representation

$$
\hat{v}_{0}(\xi)=\xi \cdot l(\xi)+h(\xi), \quad l=\left(l_{1}, \ldots, l_{n}\right),
$$

where $l$ and $h$ satisfy the following conditions:

(i) $|h(\xi)| \leq M_{0}|\xi|^{2}$ for some $M_{0}>0$;

(ii) $l$ is homogeneous of degree zero; and

(iii) $\alpha_{1}=\int_{|\omega|=1}|\omega \cdot l(\omega)|^{2} d \omega>0$.

Let $M_{1}=\sup _{|y|=1}|l(y)|, M_{2}=\sup _{\delta / 2 \leq|y| \leq 1}|\nabla l(y)|, K=\max \left(M_{0}, M_{1}, M_{2}\right)$. Then there exist constants $C_{0}$ and $C_{1}$ such that

$$
C_{0}(t+1)^{-(n / 2+1)} \leq|v(\cdot, t)|_{L^{2}}^{2} \leq C_{1}(t+1)^{-(n / 2+1)},
$$

where $C_{0}$ and $C_{1}$ both depend on $n, M_{0}, M_{1}, \delta$, and $\left|v_{0}\right|_{L^{2}}$ and $C_{0}$ also depends on $K$ and $\alpha$.

Proof. Note first that condition (iii) is not necessary for the upper bound $|v(\cdot, t)|_{L^{2}}^{2}$.

Upper bound. By Plancharel's theorem

$$
\int_{\mathbf{R}^{n}}|v|^{2} d x=\int_{\mathbf{R}^{n}}|\hat{v}|^{2} d \xi=\int_{\mathbf{R}^{n}}\left|\hat{v}_{0}\right|^{2} e^{-2|\xi|^{2} t} d \xi
$$

Let $A=\{\xi:|\xi| \leq \delta\}$. Then

$$
\begin{aligned}
\int_{\mathbf{R}^{n}}|v|^{2} d x & =\int_{A}\left|\hat{v}_{0}\right|^{2} e^{-2|\xi|^{2} t} d \xi+\int_{A^{c}}\left|\hat{v}_{0}\right|^{2} e^{-2|\xi|^{2} t} d \xi \\
& \leq \int_{A}\left|\hat{v}_{0}\right|^{2} e^{-2|\xi|^{2} t} d \xi+e^{-\delta^{2} t}\left|v_{0}\right|_{L^{2}}^{2} .
\end{aligned}
$$

To estimate the integral on the right-hand side, use the representation of $v_{0}$ in terms of $l$ and $h$.

$$
\begin{aligned}
\int_{A}\left|\hat{v}_{0}\right|^{2} e^{-2|\xi|^{2} t} d \xi= & \int_{A}|\xi \cdot l(\xi)|^{2} e^{-2|\xi|^{2} t} d \xi+2 \operatorname{Re}\left(\int_{A} \xi \cdot l(\xi) \bar{h}(\xi) e^{-2|\xi|^{2} t} d \xi\right) \\
& +\int_{A}|h(\xi)|^{2} e^{-2|\xi|^{2} t} d \xi \\
\leq & 2 \int_{A}|\xi \cdot l(\xi)|^{2} e^{-2|\xi|^{2} t} d \xi+M_{0}^{2} \int_{A}|\xi|^{4} e^{-2|\xi|^{2} t} d \xi
\end{aligned}
$$


Recall that $l(\xi)$ is homogeneous of degree zero. Combine (2.1) and (2.2) and make the change of variables $y=\sqrt{2 t} \xi$. Then

$$
\int_{\mathbf{R}^{n}}|v|^{2} d x \leq 2(2 t)^{-n / 2+1} \int_{A}|y \cdot l(y)|^{2} e^{-y^{2}} d y+M_{0}(2 t)^{-n / 2-2} \int_{A(t)}|y|^{4} e^{-y^{2}} d y,
$$

where $A(t)=\{y:|y| \leq \delta \sqrt{2 t}\}$. Since $M_{1}=\sup _{|y|=1} l(y)$ and $l$ is homogeneous of degree zero,

$$
|y \cdot l(y)|^{2} \leq|y|^{2} M_{1}^{2} .
$$

Hence from (2.3)

$$
\int_{\mathbf{R}^{n}}|v|^{2} d x \leq C /(2 t)^{n / 2+1}
$$

where $C$ depends on $M_{0}, M_{1}, \delta$, and $n$. Recall that

$$
\int_{\mathbf{R}^{n}}|v|^{2} d x \leq\left|v_{0}\right|_{L^{2}}^{2}
$$

hence

$$
\int_{\mathbf{R}^{n}}|v|^{2} d x \leq C_{1}(t+1)^{-(n / 2+1)},
$$

with $C_{1}=C_{1}\left(M_{0}, M_{1}, \delta, n,\left|v_{0}\right|_{L^{2}}\right)$.

Lower bound. Choose $\delta_{1}<\delta$ such that $4 M_{0} M_{1} \delta_{1} \leq \alpha_{1}$, and let $A_{1}=$ $\left\{\xi:|\xi| \leq \delta_{1}\right\}$. Then

$$
\begin{aligned}
\int_{\mathbf{R}^{n}}|v|^{2} d x \geq \int_{A_{1}}\left|\hat{v}_{0}\right|^{2} e^{-2|\xi|^{2} t} d \xi & \geq \int_{A_{1}}\left(|\xi \cdot l(\xi)|^{2}-2 M_{0} M_{1}|\xi|^{3}\right) e^{-2|\xi|^{2} t} d \xi \\
& =\omega_{n} \int_{0}^{\delta_{1}} r^{n+1}\left(\alpha_{1}-2 M_{0} M_{1} r\right) e^{-2 r^{2} t} d r \\
& \geq \frac{1}{2} \omega_{n} \alpha_{1} \int_{0}^{\delta_{1}} r^{n+1} e^{-2 r^{2} t} d r \\
& \geq \frac{\omega_{n} \alpha_{1} e^{-2}}{2(n+2)} t^{-(n / 2+1)} \quad \text { for } t \geq \delta_{1}^{-1} .
\end{aligned}
$$

For $t<\delta_{1}^{-2}$ we have

$$
\begin{aligned}
\int_{\mathbf{R}^{n}}|v|^{2} d x & =\int_{\mathbf{R}^{n}}\left|\hat{v}_{0}\right|^{2} e^{-2|\xi|^{2} t} d \xi \geq \int_{\mathbf{R}^{n}}\left|\hat{v}_{0}\right|^{2} e^{-2|\xi|^{2} \delta_{1}^{-2}} d \xi \\
& \geq(1+t)^{-(n / 2+1)} \int_{\mathbf{R}^{n}}\left|\hat{v}_{0}\right|^{2} e^{-2|\xi|^{2} \delta_{1}^{-2}} d \xi .
\end{aligned}
$$

The lower bound follows for

$$
C_{0}=\min \left\{\frac{\omega_{n} \alpha_{1} e^{-1}}{2(n+2)}, \int_{\mathbf{R}^{n}}\left|\hat{v}_{0}\right|^{2} e^{-2|\xi|^{2} \delta_{1}^{-2}} d \xi\right\}
$$

Corollary 2.2. Let $v$ be a solution to the heat equation with data $v_{0} \in L^{2}\left(\mathbf{R}^{n}\right)$, where $v_{0}$ has the Fourier representation described in Theorem 2.1 and $l$ and $h$ 
satisfy (i), (ii). If in addition $l$ satisfies

(1) $\omega_{0} \cdot l\left(\omega_{0}\right)=\alpha \neq 0$ for some $\omega_{0} \in S^{n-1}$ and

(2) $\xi \cdot l(\xi) \in C^{1}\left(\mathbf{R}^{n} \backslash 0\right)$,

then the conclusion of Theorem 2.1 holds.

Proof. It is necessary to show that (1) and (2) imply conditions of Theorem 2.1. Note that if $\omega_{0} \cdot l\left(\omega_{0}\right)=\alpha \neq 0$ for some $\omega_{0} \in S^{n-1}$ and $\xi \cdot l(\xi) \in C^{1}\left(\mathbf{R}^{n} \backslash 0\right)$, it follows that there exists an open ball centered at $\omega_{0}, B_{r}\left(\omega_{0}\right)$ of radius $r>0$, such that for $\omega \in B_{r}\left(\omega_{0}\right), \omega \cdot l(\omega) \geq \alpha / 2$. Hence

$$
\int_{|\omega|=1}|\omega \cdot l(\omega)|^{2} d \omega>\int_{S \cap B_{r}\left(\omega_{0}\right)}|\omega \cdot l(\omega)|^{2} d \omega \geq \alpha / 2 L>0,
$$

where $L=\int_{S \cap B_{r}\left(\omega_{0}\right)} d w$ and $S=\{\omega:|\omega|=1\}$.

The next lemma establishes an upper bound on $L^{\infty}$ rate of decay for gradient of solutions to the heat equation which decay at a given rate in $L^{2}$.

Lemma 2.3. Let $v(x, t)$ be a solution to the heat equation. Suppose $|v(\cdot, t)|_{L^{2}}^{2} \leq$ $C(t+1)^{-(n / 2+1)}$. Then

$$
|\nabla v(\cdot, t)|_{\infty}<C(t)^{-(n / 2+1)}
$$

Proof.

$$
\begin{aligned}
|\nabla v(\cdot, t)|_{\infty} & \leq|\hat{\nabla} v(\cdot, t)|_{L^{1}} \leq \int|\xi||\hat{v}(t / 2)| e^{-2|\xi|^{2} t / 2} d \xi \\
& \leq|v(\cdot, t / 2)|_{L^{2}} \cdot\left(\int|\xi|^{2} e^{-2|\xi|^{2} t} d \xi\right)^{1 / 2} \\
& =C|v(\cdot, t / 2)|_{L^{2} t^{-(n / 2+1) / 2} \leq C t^{-(n / 2+1)}} .
\end{aligned}
$$

\section{The INITIAL DATA}

In this section a class of initial data is found for which the solutions to the Navier-Stokes equations admit the Fourier representation

$$
\hat{u}_{k}\left(\xi, t_{0}\right)=\xi \cdot l_{k}\left(\xi, t_{0}\right)+h_{k}\left(\xi, t_{0}\right)
$$

for some $t_{0} \geq 0$. Here $l_{k}$ and $h_{k}$ satisfy the conditions given in Theorem 2.1. Hence the solution to the heat equation started at $u\left(x, t_{0}\right)$ has a lower bound for $L^{2}$ decay.

The initial data will belong to the intersection of $L^{1}, H^{1}$, some weighted spaces, and the complement of a set of radially equidistributed energy. The condition of not having equidistributed energy is essential in two dimensions. Specifically, an example will be given where the data has radially equidistributed energy and solution decays exponentially.

The data to be considered has Fourier transform vanishing at the origin. For nonvanishing data, the reader is referred to [4]. There are two cases.

Case 1. The zero of the origin is of order one. 
Case 2. The zero is of order greater than one.

In case one, the data can have equidistributed energy and $t_{0}$ is 0 . For case two, the data has to lie outside a set of equidistributed energy.

We define the following weighted spaces and norms.

$$
\begin{gathered}
W_{1}=\left\{u: \int_{\mathbf{R}^{n}}|x|^{2}|u| d x<\infty\right\} ; \\
W_{2}=\left\{u: \int_{\mathbf{R}^{n}}|u|^{2}|x| d x<\infty\right\} ; \\
|u|_{W_{1}}=\int_{\mathbf{R}^{n}}|x|^{2}|u| d x ; \\
|u|_{W_{2}}=\left(\int_{\mathbf{R}^{n}}|u|^{2}|x| d x\right)^{1 / 2} .
\end{gathered}
$$

Note that if $u \in W_{1} \cap W_{2} \cap L^{2}$ then $\int|x||u| d x<\infty$, since

$$
\begin{gathered}
\int_{\mathbf{R}^{n}}|x||u| d x=\int_{|x| \leq 1}|x||u| d x+\int_{|x| \geq 1}|x||u| d x \\
\leq \int_{|x| \leq 1}\left(|x|^{2}+|u|^{2}\right) d x+\int_{|x| \geq 1}|x|^{2}|u| d x<\infty . \\
V\left(\mathbf{R}^{n}\right)=C_{0}^{\infty}\left(\mathbf{R}^{n}\right) \cap\{u: \nabla \cdot u=0\}, \\
H=H\left(\mathbf{R}^{n}\right)=\text { closure of } V \text { in } L^{2} .
\end{gathered}
$$

Note that the choice of the weighted spaces insures that the data has at least two Fourier derivatives in $L^{2}$.

Theorem 3.1. Let $g \in H \cap W_{1} \cap W_{2}\left(\mathbf{R}^{n}\right), n=2,3$. If $g$ has a zero of order one at the origin, then there exists $\delta>0$ such that for $|\xi| \leq \delta$

$$
\hat{g}(\xi)=\xi \cdot l(\xi)+h(\xi),
$$

since $l$ and $h$ satisfy the hypothesis of Theorem 2.1 , with $M_{0}=\sup _{|x| \leq \delta}\left|\nabla^{2} \hat{g}(\xi)\right|$ and $\alpha$ depending only on $\widehat{\nabla g}(0)$.

Proof. Since $g \in W_{1} \cap W_{2}$,

$$
\begin{gathered}
\left|\frac{\partial}{\partial \xi_{i}} \hat{g}(\xi)\right| \leq \int|x||g| d x \leq C, \\
\left|\frac{\partial^{2}}{\partial \xi_{i} \partial \xi_{j}} \hat{g}(\xi)\right| \leq \int_{\mathbf{R}^{n}}\left|x_{i}\right|\left|x_{j}\right||g| d x \leq \int_{\mathbf{R}^{n}}|x|^{2}|g| d x \leq C .
\end{gathered}
$$

Since $\hat{g}(0)=0$ for $|\xi| \leq \delta, \delta>0$,

$$
\hat{g}(\xi)=\widehat{\nabla g}(0) \cdot \xi+\widehat{\nabla^{2}} g(\bar{\xi}) \xi^{2} .
$$

Since $\widehat{\nabla g}(0) \neq 0$ by hypothesis, to finish the proof let

$$
l(\xi)=\widehat{\nabla g}(0), \quad h(\xi)=\widehat{\nabla g}(\xi) \xi^{2} .
$$


Here $M_{0}=\sup _{|x| \leq \delta}\left|\widehat{\nabla^{2}} g(\xi)\right|$ and $\omega_{0} \in S^{n-1}$ is chosen so that $\omega_{0} \cdot \widehat{\nabla g_{k}} \neq 0$.

The following notation will be used.

$$
\begin{gathered}
\alpha_{i}^{j}\left(t_{0}, u\right)=\int_{0}^{t_{0}} \int_{\mathbf{R}^{n}}\left|u_{i}\right|^{2}-\left|u_{j}\right|^{2} d x d t, \quad \beta_{i}^{j}\left(t_{0}, u\right)=\int_{0}^{t_{0}} \int_{\mathbf{R}^{n}} u_{i} u_{j} d x d t, \\
A_{i}^{j}\left(\mathbf{R}^{n}\right)=\left\{u: \int_{\mathbf{R}^{n}}\left|u_{i}\right|^{2} d x=\int_{\mathbf{R}^{n}}\left|u_{j}\right|^{2} d x\right\}, \\
B_{i}^{j}=B_{i}^{j}\left(\mathbf{R}^{n}\right)=\left\{u: \int_{\mathbf{R}^{n}} u_{i} u_{j} d x=0\right\} .
\end{gathered}
$$

The two-dimensional case is considered first. For sake of notation, let $M=$ $A_{1}^{2} \cap B_{1}^{2}$.

Theorem 3.2. Let $g \in H^{1} \cap H \cap W_{2} \cap M^{c}\left(\mathbf{R}^{2}\right)$. Let $u(x, t)$ be a solution to the Navier-Stokes equations with data $g$. If $g$ has zero of order greater than one, then there exist $t_{0}$ and $\delta>0$ such that for $|\xi| \leq \delta$

$$
\hat{u}_{k}\left(\xi, t_{0}\right)=\xi \cdot l_{k}\left(\xi, t_{0}\right)+h_{k}\left(\xi, t_{0}\right),
$$

where $t_{0}=t_{0}\left(|g|_{H^{1}},|g|_{W_{2}}\right)$ and $l_{k}\left(\cdot, t_{0}\right)$ and $h_{k}\left(\cdot, t_{0}\right)$ satisfy

(i) $\left|h_{k}(\xi)\right| \leq M_{0}|\xi|^{2}$;

(ii) $l_{k}$ is homogeneous of degree zero;

(iii) $\omega_{0} \cdot l_{k}\left(\omega_{0}\right)=\alpha \neq 0$ for some $\omega_{0} \in S^{n-1}$ and at least one component $l_{k} ;$ and

(iv) $\boldsymbol{\xi} \cdot l_{k}(\boldsymbol{\xi}) \in C^{1}\left(\mathbf{R}^{n} \backslash(0)\right)$.

The constant $M_{0}$ depends only on $|g|_{L^{2}},|g|_{W_{2}}$, and $\delta$. The constant $\alpha$ is a multiple of $\alpha_{1}^{2}\left(t_{0}, g\right)$.

Proof. Take the Fourier transform of the Navier-Stokes equations

$$
\begin{gathered}
\hat{u}_{t}+|\xi|^{2} \hat{u}=-\widehat{u \nabla u}-\widehat{\nabla p}=-\widehat{H}, \\
\hat{u}(\xi, 0)=\hat{g}(\xi) .
\end{gathered}
$$

Hence

$$
\hat{u}_{k}(\xi, t)=\hat{g}_{k}(\xi) e^{-|\xi|^{2} t}-\int_{0}^{t} \widehat{H}_{k}(\xi, s) e^{-|\xi|^{2}(t-s)} d s .
$$

W.1.o.g. choose $k=1$. Recall that

$$
\Delta p=-\sum \frac{\partial^{2}}{\partial x_{i} \partial x_{j}} u_{i} u_{j}
$$

so that

$$
\widehat{\partial_{x_{1}} p}=-i \xi_{1} \sum_{\xi_{i} \xi_{j}} \frac{\xi_{i} \xi_{j}}{|\xi|^{2}} \widehat{u_{i} u_{j}}
$$


Let $a_{i j}={\widehat{u_{i} u_{j}}}_{\text {. Then }}$

$$
\begin{aligned}
\widehat{H}_{1}(\xi, s) & =-i\left(\sum \xi_{i} a_{i 1}-\xi_{1} /|\xi|^{2} \sum \xi_{i} \xi_{j} a_{i j}\right) \\
& =-i\left(\xi_{1}\left(1-\xi_{1}^{2} /|\xi|^{2}\right) a_{11}-\xi_{2}\left(1-2 \xi_{1}^{2} /|\xi|^{2}\right) a_{12}-\frac{\xi_{1} \xi_{2}^{2}}{|\xi|^{2}} a_{22}\right) .
\end{aligned}
$$

Note that $1-\xi_{1}^{2} /|\xi|^{2}=\xi_{2}^{2} /|\xi|^{2}$; hence

$$
\widehat{H}_{1}(\xi, s)=-i \xi \cdot\left(\xi_{2}^{2} /|\xi|^{2}\left(a_{11}-a_{22}\right),\left(1-2 \xi_{1}^{2} /|\xi|^{2}\right) a_{12}\right) .
$$

Proposition A.1 (see Appendix) establishes that for $|\xi| \leq \delta$

$$
\left|\nabla_{\xi} a_{i j}(\xi, t)\right| \leq C(t)
$$

where $C(t)$ denotes constant in $\xi$ that depends on $|g|_{L^{2}},|g|_{W_{2}}, \delta$, and $t$.

Let $a_{i j}^{0}=a_{i j}(0, t)$. Thus $a_{i j}$ can be represented as

$$
a_{i j}=a_{i j}^{0}+\xi \cdot \nabla_{\xi} a_{i j}(\bar{\xi}), \quad 0 \leq \bar{\xi} \leq \xi .
$$

By (3.3)

$$
\text { (3.5) } \begin{aligned}
\widehat{H}_{1}(\xi, s) & =-i \xi \cdot\left[\left(\xi_{2}^{2} /|\xi|^{2}\right)\left(a_{11}^{0}-a_{22}^{0}\right),\left(1-2 \xi_{1}^{2} /|\xi|^{2}\right) a_{12}^{0}+\tilde{h}_{1}(\xi, s)\right] \\
& =i \xi\left(H_{1}^{0}(\xi, s)+\tilde{h}_{1}(\xi, s)\right),
\end{aligned}
$$

and by (3.3) and (3.4)

$$
\left|\tilde{h}_{1}(\xi, s)\right| \leq C(s)|\xi|
$$

From (3.1) and (3.5)

$$
\begin{aligned}
\hat{u}(\xi, t) & =-i \xi \cdot\left(\int_{0}^{t} H_{1}^{0}(\xi, s) e^{-|\xi|^{2}(t-s)} d s+\int_{0}^{t} \tilde{h}_{1}(\xi, s) e^{-|\xi|^{2}(t-s)} d s+e^{|\xi|^{2} t} \hat{g}(\xi)\right) \\
& =\xi \cdot \int_{0}^{t}(-i) H_{1}^{0} d s+h_{1}(\xi, s)
\end{aligned}
$$

where

$$
\begin{aligned}
h_{1}(\xi, s)=-i \xi \cdot\left(\int_{0}^{t} H_{1}^{0}(\xi, s)\left(e^{-|\xi|^{2}(t-s)}-1\right) d s\right. \\
\left.\quad+\int_{0}^{t} \tilde{h}_{1}(\xi, s)^{-|\xi|^{2}(t-s)} d s+e^{-|\xi|^{2} t} \hat{g}(\xi)\right) .
\end{aligned}
$$

Since

$$
e^{-|\xi|^{2}(t-s)}-1=O\left(|\xi|^{2}\right)
$$

$\left|\xi \cdot \tilde{h}_{1}(s)\right| \leq M|\xi|^{2}$ form $|\xi| \leq \delta, M$ depending on $C(t)$;

$$
\hat{g}(\xi)=O\left(|\xi|^{2}\right) \text {, }
$$

it follows that $\left|h_{1}(\xi, t)\right| \leq M_{0}|\xi|^{2}, M_{0}$ depending only $|g|_{L^{2}}, \delta$, and $t$. Let

$$
l_{1}(\xi, t)=\left(l_{1}^{1}, l_{1}^{2}\right)=\left(-i \int_{0}^{t}\left(\xi_{2}^{2} /|\xi|^{2}\right)\left(a_{11}^{0}-a_{22}^{0}\right) d s, \int_{0}^{t}\left(1-2 \xi_{1}^{2} /|\xi|^{2}\right) a_{12}^{0} d s\right) \text {. }
$$


We first show that for some $t_{0}>0$ and some $\omega_{0} \in S^{2}, \omega_{0} \cdot l_{1}\left(\omega_{0}, t_{0}\right) \neq 0$. If $u_{0} \notin A_{1}^{2}$ choose $\omega_{0}=(1,1) / \sqrt{2}$; then

$$
\omega_{0} \cdot l_{1}\left(\omega_{0}, t_{0}\right)=\frac{-i}{2 \sqrt{2}} \int_{0}^{t_{0}} \int_{\mathbf{R}^{2}} u_{1}^{2}-u_{2}^{2} d x d y d s .
$$

If $u_{0} \notin B_{1}^{2}$ choose $\omega_{0}=(1,0)$; then

$$
\omega_{0} \cdot l_{1}\left(\omega_{0}, t_{0}\right)=\int_{0}^{t_{0}} \int_{\mathbf{R}^{2}} u_{1} u_{2} d x d y d s
$$

By Lemmas A.2 and A.3 of the appendix since $g \in M^{c}$ there exists $t_{0}=$ $t_{0}\left(|g|_{L^{2}},|g|_{W_{2}}, \alpha\right)$ such that RHS of (3.6) and (3.7) are not zero. Hence

$$
\omega_{0} \cdot l_{1}\left(\omega_{0}, t_{0}\right) \neq 0
$$

Conditions (i), (ii), and (iv) are trivially satisfied for $l_{1}\left(\xi, t_{0}\right)$ and $h_{1}\left(\xi, t_{0}\right)$.

Theorem 3.3. Let $g \in H \cap W_{2}\left(\mathbf{R}^{3}\right)$. Let $u(x, t)$ be a suitable Leray-Hopf solution in the sense of Caffarelli, Kohn, and Nirenberg with data $g$. Let $g$ have a zero at the origin of order greater than one. If there exists $t_{0}$ such that

or

$$
\alpha_{i}^{j}\left(t_{0}, 3\right)=\int_{0}^{t_{0}} \int_{\mathbf{R}^{3}}\left|u_{i}\right|^{2}-\left|u_{j}\right|^{2} d x d t \neq 0, \quad i, j=1,2,3
$$

$$
\beta_{i}^{j}\left(t_{0}, 3\right)=\int_{0}^{t_{0}} \int_{\mathbf{R}^{3}} u_{i} u_{j} d x d t \neq 0, \quad i \neq j,
$$

then there exists $\delta>0$ such that for $|\xi| \leq \delta$,

$$
\hat{u}_{k}\left(\xi, t_{0}\right)=\xi \cdot l_{k}\left(\xi, t_{0}\right)+h_{k}\left(\xi, t_{0}\right) \text {, }
$$

where $l_{k}\left(\xi, t_{0}\right)$ and $h_{k}\left(\xi, t_{0}\right)$ satisfy the same conditions as in Theorem 3.2.

Proof. Recall that a weak solution with data $g$ satisfies

$$
\begin{aligned}
\langle u(t), \phi(t)\rangle-\int_{0}^{t}\left\{\left\langle u(s) \frac{\partial}{\partial s} \phi(s)\right\rangle\right. & +\langle\nabla u(s), \nabla \phi(s)\rangle \\
& +\langle(u(s) \cdot \nabla) u(s), \phi(s)\rangle\} d s-\langle g, \phi(0)\rangle=0
\end{aligned}
$$

for all smooth vectors $\phi$ with compact support and $\operatorname{div} \phi=0$. Following Wiegner's argument [6], we choose $\phi$ to be the solution to the heat equation with data $\phi_{0} \in C_{0}^{\infty}\left(\mathbf{R}^{3}\right)$ and $\operatorname{div} \phi_{0}=0$. This $\phi$ is smooth and bounded in $L^{\infty}$ and (3.8) holds for $\phi$ by approximation. Let $t_{0}>0$ be fixed and $t^{*}>t_{0}$. For $0 \leq s \leq t$ let

$$
\phi(s)=F^{-1}\left(F\left(\phi_{0}\right) \exp \left(-|\xi|^{2}, t^{*}-s\right)\right),
$$

which is the solution to the homogeneous heat system with data $\phi_{0}$ at time $t^{*}-s$. It is easy to show that for such a choice of $\phi,(3.8)$ yields

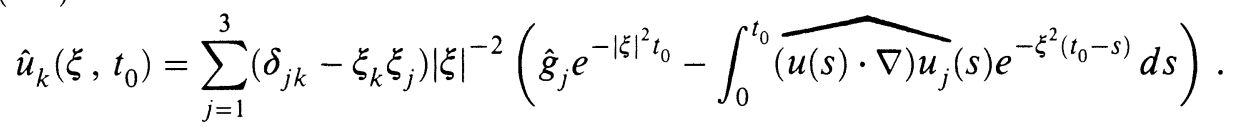


For more details we refer the reader to [6]. By hypothesis

$$
\hat{g}_{j}(\xi)=\hat{g}_{j}(0)+\widehat{\nabla g}_{j}(0) \cdot \xi+\widehat{\nabla}^{2} g_{j}(\bar{\xi})(\xi, \xi)=\widehat{\nabla}^{2} g_{j}(\bar{\xi})(\xi, \xi) \text {. }
$$

Hence we only have to consider the terms in

$$
\sum_{j=1}^{3}\left(\delta_{k j}-\xi_{k} \xi_{j}\right)|\xi|^{-2} \int_{0}^{t_{0}} \widehat{u \nabla u_{j}}(s) e^{-|\xi|^{2}\left(t_{0}-s\right)} d s .
$$

By Note A.4 (see Appendix) the last expression can be rewritten as

$$
-i \sum_{j=1}^{3}\left(\delta_{k j}-\xi_{k} \xi_{j}\right)|\xi|^{-2} \int_{0}^{t_{0}} \sum_{i, j=1}^{3} \xi_{i} a_{i j}^{0}(s) d s+h(\xi),
$$

where $|h(\xi)| \leq K|\xi|^{2}$ with $K$ depending only on the $L^{2}, W_{2}$ norms of the data, and $t_{0}$. The vector function $l_{k}=\left(l_{k}^{1}, l_{k}^{2}, l_{k}^{3}\right)$ can be chosen so that the components have the form

$$
l_{k}^{i}=-i \sum_{j=1}^{3}\left(\delta_{k j}-\left(\xi_{k} \xi_{j}\right)\right)|\xi|^{-2} \int_{0}^{t_{0}} \sum_{i, j=1}^{3} a_{i j}^{0}(s) d s .
$$

From (3.9) and (3.10) it follows that

$$
\hat{u}_{k}\left(\xi, t_{0}\right)=\xi \cdot l_{k}\left(\xi, t_{0}\right)+h_{k}\left(\xi, t_{0}\right)
$$

with $\left|h_{k}\left(\xi, t_{0}\right)\right| \leq K_{0}|\xi|^{2}$, and $K_{0}$ depends only on $\sup _{|\xi| \leq \delta}\left|\widehat{\nabla^{2}} g(\xi)\right|$, the $L^{2}, L_{W_{2}}$ norms of $g$ and $t_{0}$. Conditions (i), (ii), and (iv) of Theorem 3.2 follow trivially. To establish (iii) choose $\omega_{0}=(1 / \sqrt{2})\left(e_{i}+e_{j}\right)$ if $\alpha_{i}^{j} \neq 0$ with $e_{i}$ the $i$ th element of the canonical basis of $\mathbf{R}^{3}$. If $\beta_{i}^{j} \neq 0$

$$
\text { let } \omega_{0}=e_{j} \text { if } j \neq k \text { or } \omega_{0}=e_{i} \text { if } i \neq k \text {. }
$$

\section{THE KEY THEOREM}

The decay rates for $L^{2}$ norms of solutions to the heat equation are compared to the decay rates of $L^{2}$ norms of solutions to the Navier-Stokes equations. It is shown that if the upper bounds and lower bounds of decay rates of solutions to the heat equation are of order $(t+1)^{-(n / 4+1 / 2)}$, then the same rates are valid for solutions to the Navier-Stokes equations starting with the same data.

A formal argument is given for solutions in $n$ dimensions, $n \geq 2$. The proof is rigorous for $n=2$. For $n=3$, the rigorous results are less strong, since they are obtained only under the supposition of the existence of a sequence of approximate solutions (such as those constructed by Caffarelli, Kohn, and Nirenberg [1]) which converge strongly in $L^{2}\left([0,1] \times \mathbf{R}^{3}\right)$. Passing to the limit a lower bound will be obtained for almost all $t$. We expect that the result for $n=3$ can be improved. For $n>3$ it is expected that the proof can be applied 
to the approximate solutions constructed by Kayikiya and Miyakawa [2] and passing to the limit. The upper limits of the rates of decay are included for completeness. The bounds for $n=2$ were established by Wiegner in [6]. The bounds for $n=3$ were established in [5, 6].

The proof for the lower bound is based on an analysis of the Fourier transform of the difference between the solutions to the heat equation and the NavierStokes equations starting with the same data. The argument is by contradiction.

Theorem 4.1. Let $u_{0} \in L^{1} \cap W_{2} \cap H\left(\mathbf{R}^{n}\right), n=2$, 3. Let $v$ be a solution to the heat equation with data $u_{0}$. Suppose

$$
C_{0}(1+t)^{-(n / 2+1)} \leq|v(\cdot, t)|_{L^{2}}^{2} \leq C_{1}(1+t)^{-(n / 2+1)} .
$$

For $n=2$ let $u(\cdot, t)$ be a solution to the Navier-Stokes equations with data $u_{0}$. Then there exist constants $M_{0}$ and $M_{1}$ such that

$$
M_{0}(1+t)^{-(n / 2+1)} \leq|u(\cdot, t)|_{L^{2}}^{2} \leq M_{1}(1+t)^{-(n / 2+1)},
$$

where $M_{0}$ and $M_{1}$ depend on $C_{1}, n$, and the $L^{1}$ and $L^{2}$ norms of $u_{0}$, and $M_{0}$ depends also on $C_{0}$ and the $W_{2}$ norm of $u_{0}$.

For $n=3$, the upper bound in (4.2) holds for all $t$ and the lower bound holds for a.e. $t$, where $u(x, t)$ is a Leray-Hopf solution in the sense of Caffarelli, Kohn, and Nirenberg.

Proof.

Upper bound. See [5, 6].

Lower bound. We first present a proof that is rigorous for $n=2$ and formal for $n \geq 3$. We will indicate the modifications necessary to make the proof rigorous for $n=3$.

Outline of the proof. There are two cases to consider. Let $\beta$ be a fixed constant, which will be defined below. For $n=2$ the cases are the following.

Case 1. Given $t$ there exists $T>t$ such that

$\left.\left|\int_{0}^{T} \int_{\mathbf{R}^{n}}\right| u_{1}\right|^{2}-\left|u_{2}\right|^{2} d x d x \mid<\beta \sqrt{C_{0}}$ and $\left|\int_{0}^{T} \int_{\mathbf{R}^{n}} u_{1} u_{2} d x d s\right|<\beta \sqrt{C_{0}}$.

Case 2. There exists $T_{0}$ such that for all $t \geq T_{0}$

$$
\left.\left|\int_{0}^{t} \int_{\mathbf{R}^{n}}\right| u_{1}\right|^{2}-\left|u_{2}\right|^{2} d x d s \mid \geq \beta \sqrt{C_{0}} \text { or }\left|\int_{0}^{t} \int_{\mathbf{R}^{n}} u_{1} u_{2} d x d s\right| \geq \beta \sqrt{C_{0}} \text {. }
$$

If $n>2$ the two cases are slightly different.

Case $1^{*}$. Given $t$ there exists $T>t$ such that

$$
\left.\left|\int_{0}^{T} \int_{\mathbf{R}^{n}}\right| u_{i}\right|^{2}-\left|u_{j}\right|^{2} d x d s \mid<\beta \sqrt{C_{0}}, \quad 1 \leq i, j \leq n
$$

and

$$
\left|\int_{0}^{T} \int_{\mathbf{R}^{n}} u_{i} u_{j} d x d s\right|<\beta \sqrt{C_{0}}, \quad 1 \leq i, j \leq n, i \neq j .
$$


Case $2^{*}$. There exists $T_{0}$ such that for all $t \geq T_{0}$

$$
\left.\left|\int_{0}^{t} \int_{\mathbf{R}^{n}}\right| u_{i}\right|^{2}-\left|u_{j}\right|^{2} d x d s \mid \geq \beta \sqrt{C_{0}} \text { for at least one pair } i, j
$$

or

$$
\left|\int_{0}^{t} \int_{\mathbf{R}^{n}} u_{i} u_{j} d x d s\right| \geq \beta \sqrt{C_{0}} \text { for at least one pair } i, j \text { with } i \neq j .
$$

Outlines of the proofs of Cases 1 and 2 are given first followed by a detailed proof of each one.

Outline of Case 1. In this case an increasing sequence $\left\{r_{m}\right\}, r_{m}=r_{m}(\beta)$, $r_{m} \rightarrow \infty$ as $m \rightarrow \infty$, can be constructed such that

$$
\left.\left|\int_{0}^{r_{m}} \int_{\mathbf{R}^{n}}\right| u_{1}\right|^{2}-\left|u_{2}\right|^{2} d x d s \mid<\beta \sqrt{C_{0}} \text { and }\left|\int_{0}^{r_{m}} \int_{\mathbf{R}^{n}} u_{1} u_{2} d x d s\right|<\beta \sqrt{C_{0}} .
$$

Let $\omega=v-u$ be the difference of the solution to the heat equation and the solution to the Navier-Stokes equations with the same data $u_{0}$. It will be shown first that for $t$ sufficiently large

$$
|\omega(\cdot, t)|_{L^{2}}^{2}<C(t+1)^{-2 \alpha}+O\left((t+1)^{-\gamma}\right),
$$

where $\alpha=n / 4+1 / 2, \gamma>2 \alpha$, and $C \leq C_{0} / 4$. Briefly, inequality (4.4) is obtained as follows. The difference $\omega$ can be considered as a solution to an inhomogeneous heat equation

$$
\omega_{t}=\Delta \omega+A,
$$

where $A=-(u \nabla u+\nabla p)$. Using the Fourier splitting method [4, 5], it follows that the $L^{2}$ norm of $\omega$ is bounded by three terms. Two of these terms come from the inhomogeneous part and decrease at a faster rate than $\alpha$. This faster decay is a consequence of the terms either being cubic in $(\omega, u)$ or involving the gradient $v$, which improves the decay rate. The third term is the critical one and has the form

$$
\int_{S(t)}|\hat{\omega}(\xi, t)|^{2} d \xi
$$

where $S(t)$ is a ball of radius $(t+1)^{-n / 2}$ and as such decays like $(t+1)^{-n / 2}$. The hypothesis of Case 1 is used (or Case $1^{*}$ if $n>2$ ) to show that

$$
|\hat{\omega}(\xi, t)| \leq\left[2 \beta \sqrt{C_{0}}+C_{2}\left(1+r_{m}\right)^{-n}\right]|\xi|+O\left(|\xi|^{2}\right),
$$

where $s \geq 2$ and $C_{2}$ is an appropriate constant. Hence

$$
\int_{S(t)}|\hat{\omega}(\xi, t)|^{2} d \xi \leq\left[8 \beta^{2} C_{0}+2 C_{2}\left(1+r_{m}\right)^{-2 n}\right](t+1)^{-(n / 2+1)}+O\left(t^{-\alpha}\right),
$$

where $\alpha>n / 2+1$. Here $C_{2}$ and $C_{3}$ depend on the $L^{2}$ and $L^{1}$ norms of $u_{0}$. Choosing $\beta$ sufficiently small and $r_{m}$ sufficiently large, (4.4) will yield for $t \geq T_{0}, T_{0}=T_{0}\left(\left|u_{0}\right|_{L^{2}},\left|u_{0}\right|_{L^{1}}, n, C_{0}, C_{1}\right)$

$$
|\omega(\cdot, t)|_{L^{2}}^{2} \leq C_{0} / 4(t+1)^{-(n / 2+1)} .
$$


Hence the lower bound of $|u(\cdot, t)|_{L^{2}}$ follows for $t \geq T_{0}$. For $t \leq T_{0}$ the bound is a consequence of the decay of energy of $u$.

Outline of Case 2. Here we study the difference $W=U-V$ where $U=$ $u(x, t+T)$ with $T>T_{0}$ for some appropriate $T$ and $V$ is the solution to the heat equation

$$
\begin{gathered}
V_{t}=\Delta V, \\
V(x, 0)=u(x, T) .
\end{gathered}
$$

By Theorems 3.2 and 2.1 and the hypothesis of Case 2 (or Case $2^{*}$ if $n>2$ ) the $L^{2}$ norm of $V$ has the following upper and lower bounds:

$$
C_{0} \beta_{1}(t+1)^{-(n / 2+1)} \leq|V(\cdot, t)|_{L^{2}}^{2} \leq K_{1}(t+1)^{-(n / 2+1)}
$$

for some appropriate constant $\beta_{1}$. As in Case $1, W$ satisfies an inhomogeneous heat equation. Again using the Fourier splitting method, the critical term to bound is

$$
\int_{S(t)}|\widehat{W}(\xi, t)|^{2} d \xi
$$

Hypothesis of Case 2 yields

$$
|\widehat{W}(\xi, t)| \leq C(T+1)^{-n / 2}|\xi|,
$$

and as in Case 1, if $T$ sufficiently large and $t>T$, it will follow that

$$
\int_{\mathbf{R}^{n}}|\widehat{W}(\xi, t)|^{2} d x \leq C(T+1)^{-n / 2}(t+1)^{-(n / 2+1)} \leq \frac{C_{0} \beta_{1}}{4}(t+1)^{-(n / 2+1)} .
$$

The details of the proof are given next. We give the proof for $n=2$; if $n>2$ the hypotheses are given by Cases $1^{*}$ and $2^{*}$ and (3.5) needs to be modified appropriately.

Detailed proofs of Cases 1 and 2 follow.

Proof of Case 1. Let $\left\{r_{k}\right\}$ be an increasing sequence such that (4.3) holds. Let $\omega=v-u$ be the difference between the solution to the heat equation and the solution to the Navier-Stokes. Then

$$
\omega_{t}=\Delta \omega-(u \nabla u+\nabla p) .
$$

Multiplying (4.5) by $\omega$ and integrating in space yields, after some integration by parts,

$$
\frac{d}{d t} \int_{\mathbf{R}^{n}} \frac{|\omega|^{2}}{2} d x=-\int_{\mathbf{R}^{n}}|\nabla \omega|^{2} d x-\int_{\mathbf{R}^{n}}(u-v) u \nabla u d x-\int_{\mathbf{R}^{n}} \omega \nabla p d x .
$$

Since $\operatorname{div} \omega=0$, the last integral vanishes. Moreover, since $\operatorname{div} u=0$, 
$\int u u \nabla u d x=0$. Hence

$$
\begin{aligned}
\frac{d}{d t} \int_{\mathbf{R}^{n}}|\omega|^{2} d x & =2 \int_{\mathbf{R}^{n}}|\nabla \omega|^{2} d x-2 \sum_{j=1}^{n} \int_{\mathbf{R}^{n}} v_{j} \sum_{i=1}^{n} \frac{\partial}{\partial x_{i}}\left(u_{i} u_{j}\right) d x \\
& =-2 \int_{\mathbf{R}^{n}}|\nabla \omega|^{2} d x+2 \sum_{j=1}^{n} \int_{\mathbf{R}^{n}}\left(u_{i} u_{j}\right) \frac{\partial}{\partial x_{i}} v_{j} d x \\
& \leq-2 \int_{\mathbf{R}^{n}}|\nabla \omega|^{2} d x+K|\nabla v|_{\infty} \int_{\mathbf{R}^{n}}|u|^{2} d x,
\end{aligned}
$$

where $K=2 n^{2}$. Using Plancherel's theorem the last equality reads

$$
\frac{d}{d t} \int_{\mathbf{R}^{n}}|\hat{\omega}|^{2} d \xi \leq-2 \int_{\mathbf{R}^{n}}|\xi|^{2}|\hat{\omega}|^{2} d \xi+K|\nabla v|_{\infty} \int_{\mathbf{R}^{n}}|u|^{2} d x
$$

Let

$$
S(t)=\left\{\xi:|\xi| \leq\left(\frac{2 n}{t+1}\right)^{1 / 2}\right\}
$$

Splitting the frequency domain to sets $S(t)$ and $S(t)^{c}$, arguments of previous work done in [5], yields

$$
\begin{aligned}
\frac{d}{d t}\left((t+1)^{4 n} \int_{\mathbf{R}^{n}}|\omega|^{2} d x\right) & \leq(t+1)^{4 n-1}\left[4 \int_{S(t)}|\hat{\omega}|^{2} d \xi+K|\nabla v|_{\infty} \int_{\mathbf{R}^{n}}|u|^{2} d x\right] \\
& =I_{1}+I_{2} .
\end{aligned}
$$

Consider first $I_{2}$. By Lemma 2.1

$$
I_{2} \leq K C_{1} t^{-n / 2-1}(t+1)^{4 n-1} \int_{\mathbf{R}^{n}}|u|^{2} d x .
$$

Recall that by the results of Wiegner [6] there is a constant $C$ depending only on the norms of the initial data such that

$$
|\omega|_{L^{2}}^{2} \leq C(t+1)^{-(n / 2+1)} \text {. }
$$

Since

$$
|v|_{L^{2}}^{2} \leq C(t+1)^{-(n / 2+1)},
$$

it follows that if $C_{2}=C+C_{1}$ then

$$
|u(\cdot, t)|_{L^{2}}^{2} \leq C_{2}(t+1)^{-(n / 2+1)} .
$$

Hence by (4.8) and Lemma 2.1 there is a constant $M$ such that for $t \geq 1$

$$
I_{2} \leq M(t+1)^{3 n-3}
$$

where $M_{1}=K C_{1} C_{2}$. To bound integral $I_{1}$ the following estimates on $\hat{\omega}(\xi, t)$ are necessary. Taking the Fourier transform on the equations for the difference yields

$$
\begin{gathered}
\hat{\omega}_{k, t}(\xi, t)+|\xi|^{2} \hat{\omega}_{k}=-\widehat{u \nabla u_{k}}-\widehat{\nabla_{k} p}=-\widehat{H}_{k}, \quad k=1,2 ; \\
\hat{\omega}_{k}(\xi, 0)=0 .
\end{gathered}
$$


For sake of notation, let $\omega_{k}=\omega, H_{k}=H$. Since

$$
\widehat{H}=\widehat{u \nabla u}-\widehat{\nabla p}=-i\left[\sum_{j=1}^{n} \xi_{j}{\widehat{u_{j} u_{k}}}_{-\xi_{k}} \sum_{i, j} \frac{\xi_{r} \xi_{j}}{|\xi|^{2}}{\widehat{u_{r}}}_{j}\right],
$$

it follows that

$$
|\widehat{H}(\xi, t)| \leq K_{1}|\xi||u(\cdot, t)|_{L^{2}}^{2}
$$

with $K_{1}=n(n+1)$. Hence for $t \geq r_{m}$, where $r_{m}$ will be chosen below,

$$
|\hat{\omega}(\xi, t)| \leq\left|\hat{\omega}\left(\xi, r_{m}\right)\right| e^{-|\xi|^{2}\left(t-r_{m}\right)}+K_{1}|\xi| \int_{r_{m}}^{t} e^{-|\xi|^{2}(t-s)}|u(\cdot, s)|_{L^{2}}^{2} d s=\mathrm{I}+\mathrm{II} \text {. }
$$

Bound for I. Hypothesis for Case 1 (Case $1^{*}$ if $n>2$ ) is used to show that $\hat{\omega}\left(\xi, r_{m}\right)$ is sufficiently small. Then $r_{m}$ is chosen so large that the decay of the energy will imply that the coefficient of II is sufficiently small. Since $r_{m}$ was chosen so that

$$
\left.\left|\int_{0}^{r_{n}} \int_{\mathbf{R}^{2}}\right| u_{1}\right|^{2}-\left|u_{2}\right|^{2} d x \mid \leq \beta \sqrt{C_{0}}
$$

and

$$
\left|\int_{0}^{r_{m}} \int_{\mathbf{R}^{2}} u_{1} u_{2} d x\right| \leq \sqrt{C_{0}} \beta
$$

and by $(3.5)$

$$
\hat{H}(\xi, s)=i \xi \cdot\left(\frac{\xi_{2}^{2}}{|\xi|^{2}}\left(a_{11}^{0}-a_{22}^{0}\right), 1-\frac{2 \xi_{1}^{2}}{|\xi|^{2}} a_{12}^{0}\right)+O\left(|\xi|^{2}\right)
$$

(Recall that if $n>2, \widehat{H}$ will be modified appropriately.) It follows that

$$
\left|\hat{\omega}\left(\xi, r_{m}\right)\right| \leq \int_{0}^{r_{m}}|\widehat{H}(\xi, s)| d s \leq 2 \beta \sqrt{C_{0}}|\xi|+O\left(|\xi|^{2}\right) r_{m} .
$$

Bound for II. From (4.8)

$$
\begin{aligned}
\mathrm{II} & \leq K_{1}|\xi| C_{2} \int_{r_{m}}^{t} \frac{1}{(1+s)^{n / 2+1}} d s \\
& \leq \frac{1}{n / 2+2} K_{1}|\xi| C_{2}\left(1+r_{m}\right)^{-n / 2} \leq 2 n\left(1+r_{m}\right)^{-n / 2} C_{2}|\xi| .
\end{aligned}
$$

Hence

$$
\begin{aligned}
I_{1} & \leq(t+1)^{4 n-1} \int_{S(t)}[\mathrm{I}+\mathrm{III}]^{2} d \xi \\
& \leq 4 n(t+1)^{4 n-1}\left[4 \beta^{2} C_{0} 2 \int_{S(t)}|\xi|^{2} d \xi+4 n^{2}\left(1+r_{m}\right)^{-n} C_{2}^{2} \int_{S(t)}|\xi|^{2} d \xi\right. \\
& \leq(t+1)^{4 n-1} \frac{C_{0}}{8}(t+1)^{-n / 2-1}+O\left((t+1)^{(7 / 2) n-3}\right),
\end{aligned}
$$


where we let $\beta$ be so that $16 \beta^{2} A_{n}=1 / 16$ with $A_{n}=2 \pi(2 n)^{n / 2+1} /(n+2)$ and then choose $r_{m}$ so that $A_{n} 16 n^{2}\left(1+r_{m}\right)^{-n} C_{2}^{2} \leq C_{0} / 16$. Integrate (4.6) over $[1, t]$ and use the bounds for $I_{1}$ and $I_{2}$ to obtain

$$
\begin{aligned}
\int_{\mathbf{R}^{n}}|\omega(x, t)|^{2} d x \leq & (t+1)^{-4 n} \int_{\mathbf{R}^{n}}|\omega(x, 1)|^{2} d x+M(t+1)^{-(n+2)} \\
& +\frac{C_{0}}{8}(t+1)^{-(n / 2+1)}+\text { H.O.T. }
\end{aligned}
$$

Recall that

$$
|\omega(x, 1)|_{L^{2}}^{2} \leq|u(\cdot, 1)|_{L^{2}}^{2}+|v(\cdot, 1)|_{L^{2}}^{2} \leq 2\left|u_{0}\right|_{L^{2}}^{2} .
$$

Hence (4.10) implies

$$
\int_{\mathbf{R}^{n}}|\omega(x, t)|^{2} d x \leq \frac{C_{0}}{8}(t+1)^{-(n / 2+1)}+\text { H.O.T. }
$$

By (3.5)

$$
\begin{aligned}
\text { H.O.T. }= & {\left[\int_{0}^{t}(s+1)^{4 n-1} \int_{S(t)} O\left(|\xi|^{4}\right) d \xi d s r_{m}\right](t+1)^{-4 n} } \\
& +M(t+1)^{-(n+2)}+2\left|u_{0}\right|_{L^{2}}^{2}(t+1)^{-4 n} \\
\leq & C\left(r_{m},\left|u_{0}\right|_{W_{2}},\left|u_{0}\right|_{L^{1}},\left|u_{0}\right|_{L^{2}}\right)(t+1)^{-\sigma},
\end{aligned}
$$

where $\sigma \geq n / 2+2$. Hence for $t \geq T_{0}$ with $T_{0}$ depending only on $r_{m},\left|u_{0}\right|_{L^{1}}$, $\left|u_{0}\right|_{L^{2}},\left|u_{0}\right|_{W_{2}}$, and $C_{0}$,

$$
\int_{\mathbf{R}^{n}}|\omega(x, t)|^{2} d x \leq \frac{C_{0}}{4}(t+1)^{-(n / 2+1)} .
$$

Thus for $t \geq T_{0}$

$$
|u(\cdot, t)|_{L^{2}}=|v|_{L^{2}}-|\omega|_{L^{2}} \geq \sqrt{C_{0}} / 2(t+1)^{-n / 2-1} .
$$

If $t<T_{0}$ then

$$
\begin{aligned}
|u(\cdot, t)|_{L^{2}}^{2} & \geq\left|u\left(\cdot, T_{0}\right)\right|_{L^{2}}^{2} \geq C_{0} / 4\left[\frac{t+1}{T_{0}+1}\right]^{n / 2+1}\left[\frac{1}{t+1}\right]^{n / 2+1} \\
& \geq \frac{C_{0}}{4}\left(T_{0}+1\right)^{-(n / 2+1)}(t+1)^{-(n / 2+1)}
\end{aligned}
$$

and this proves Case 1 since $T_{0}$ depends only on $\left|u_{0}\right|_{L^{1}},\left|u_{0}\right|_{L^{2}},\left|u_{0}\right|_{W_{2}}, n$, and $C_{0}$.

The following auxiliary computations will be needed for Case 2 .

1. Lower bound for $\alpha_{1}$. Recall from Theorems 2.1 and 3.2 that

$$
\alpha_{1}=\int_{|\omega|=1}\left|\omega \cdot l_{1}(\omega)\right|^{2} d \sigma
$$


where

$$
\begin{gathered}
l_{1}(\omega, t)=\left(-i \int_{0}^{t}\left(\omega_{2}^{2} /|\omega|^{2}\right)\left(a_{11}^{0}-a_{22}^{0}\right) d s, \int_{0}^{t}\left(1-2 \omega_{1}^{2} /|\omega|^{2}\right) a_{12}^{0} d s\right), \\
a_{i j}^{0}=\int_{\mathbf{R}^{n}} u_{i} u_{j} d x .
\end{gathered}
$$

Hence

$$
\alpha_{1}=\int_{|\omega|=1} \omega_{1}^{2} \omega_{2}^{4}\left(\int_{0}^{t} a_{11}^{0}-a_{22}^{0} d s\right)+\left(1-2 \omega_{1}^{2}\right) \omega_{2}^{2}\left(\int_{0}^{t} a_{12} d s\right)^{2} d \sigma
$$

Since by hypothesis of Case 2 for $t \geq T_{0}$,

$$
\begin{gathered}
\int_{0}^{t} a_{11}^{0}-a_{22}^{0} d s=\int_{0}^{t} \int_{\mathbf{R}^{n}} u_{1}^{2}-u_{2}^{2} d x d s \geq \beta \sqrt{C_{0}}, \\
\int_{0}^{t} a_{12}^{0} d s=\int_{0}^{t} \int_{\mathbf{R}^{n}} u_{1} u_{2} d x d s \geq \beta \sqrt{C_{0}},
\end{gathered}
$$

it follows that

$$
\alpha_{1} \geq \beta^{2} C_{0} \int_{|\omega|=1} \omega_{1}^{2} \omega^{4}+\left(1-2 \omega_{1}^{2}\right)^{2} \omega_{2}^{2} d \sigma=\beta^{2} C_{0} \gamma
$$

2. Lower bound for the solution to heat equation $V(x, t)$ with data $u\left(x, T_{1}\right)$, $T_{1} \geq T_{0}, T_{0}$ given by hypothesis of Case 2. By Theorems 2.1 and 3.2

$$
\hat{u}_{1}\left(\xi, T_{1}\right)=\xi \cdot l_{1}\left(\xi, T_{1}\right)+h_{1}\left(\xi, T_{1}\right)
$$

and $|V(x, t)|_{L^{2}}$ satisfies for $t \geq \delta_{1}, \delta_{1}=\left(\delta\left(T_{1}\right)\right)^{-1}\left(\right.$ where $\left.4 \delta M_{0} M_{1} \leq \alpha_{1}\right)$,

$$
|V(\cdot, t)|_{L^{2}}^{2} \geq \chi_{0}(t+1)^{-(n / 2+1)}
$$

where $\chi_{0}=\alpha_{1} \omega_{n} e^{-1} / 2(n+2)$. By the computations for $\alpha_{1}$ it follows that

$$
\chi_{0} \geq \beta^{2} C_{0} \gamma \omega_{n} e^{-1} / 2(n+2)=\chi_{1} .
$$

Hence for $t \geq \delta=\delta_{1}\left(T_{1}\right)$

$$
|V(\cdot, t)|_{L^{2}}^{2} \geq \chi_{1}(t+1)^{-(n / 2+1)},
$$

and $\chi_{1}$ is independent of $T_{1}$.

Proof of Case 2. Let $V$ be the solution to the heat equation with data $V(x, 0)=u\left(x, T_{1}\right)$, for $T_{1}$ satisfying

(i) $T_{1}>T_{0}, T_{0}$ given by hypothesis of Case 2 ;

(ii) $4 n^{2} C^{2}\left(1+T_{1}\right)^{-n} \leq \chi_{1} A_{n}^{-1} / 8$,

where $\chi_{1}$ is given by the auxiliary computations 1 . The constant $C$ is such that $|u(\cdot, t)|_{L^{2}}^{2} \leq C(t+1)^{-(n / 2+1)}$ and $A_{n}=2 \pi(2 n)^{n / 2+1} /(n+2)$. Thus by Theorems 2.1 and 3.2 it follows that for $t \geq \delta_{1}=\delta\left(T_{1}\right)^{-1}$

$$
\chi_{1}(t+1)^{-(n / 2+1)} \leq|V(\cdot, t)|_{L^{2}}^{2} \leq K_{0}(t+1)^{-(n / 2+1)},
$$


where $K_{0}$ depends on the $L^{2}$ norm of $u_{0}$ and $\chi_{1}$ depends on $\beta$ and $C_{0}$. Note that $\chi_{1}$ is independent of $T_{1}$, but if $T_{1}$ tends to infinity then the lower bound of (4.12) is only valid for $t \geq \delta_{1}=\delta(T)^{-1}$ and $\delta\left(T_{1}\right)^{-1}$ tends to infinity as $T_{1}$ tends to infinity. Let

$$
U(x, t)=u\left(x, t+T_{1}\right) .
$$

We study the difference $W=V-U$. Here the hypothesis of Case 2 (Case $2^{*}$ if $n>2)$ together with the decay of energy of the solutions to Navier-Stokes will imply that $|W(\cdot, t)|_{L^{2}}^{2} \leq C(t+1)^{-(n / 2+1)}$ with $C$ sufficiently small. As in Case $1, W$ satisfies an inhomogeneous heat equation. The Fourier splitting method will yield

$$
\frac{d}{d t}\left[(t+1)^{4 n} \int_{\mathbf{R}^{n}}|W|^{2} d x\right] \leq(t+1)^{4 n-1} \int_{S(t)}|\widehat{W}|^{2} d \xi+K|\nabla V|_{\infty} \int_{\mathbf{R}^{n}}|u|^{2} d x .
$$

The second term can be bounded as in Case 1 for $t \geq 1$

$$
K|\nabla V|_{\infty} \int_{\mathbf{R}^{n}}|U|^{2} d x \leq M(t+1)^{3(n-1)} .
$$

To bound the first term note that the Fourier transform of the equation for $W$ yields

$$
\widehat{W}+|\xi|^{2} \widehat{W}=-U \nabla U-\nabla P=\widehat{H},
$$

where $P(x, t)=p\left(x, t+T_{1}\right)$. As Before $|\widehat{H}(\xi, t)| \leq K_{1}|\xi||U(\cdot, t)|_{L^{2}}^{2}$; hence

$$
\begin{aligned}
|\widehat{W}(\xi, t)| & \leq \int_{0}^{t}|\widehat{H}(\xi, s)| d s \leq K_{1}|\xi| \int_{0}^{t}|U(x, s)|_{L^{2}}^{2} d s \\
& =K_{1}|\xi| \int_{0}^{t}\left|u\left(x, s+T_{1}\right)\right|_{L^{2}}^{2} d s=K_{1}|\xi| \int_{T_{1}}^{t+T_{1}}|u(x, s)|_{L^{2}}^{2} d s \\
& \leq C K_{1}|\xi| \int_{T_{1}}^{t+T_{1}}\left[\frac{1}{1+s}\right]^{n / 2+1} d s \leq 2 n C|\xi|\left[\frac{1}{1+T_{1}}\right]^{n / 2} \\
& =2 n C|\xi|\left(\frac{1}{1+T_{1}}\right)^{n / 2} .
\end{aligned}
$$

Hence the first term in (4.13) can be bounded as follows:

$$
\begin{aligned}
(t+1)^{4 n-1} \int_{S(t)}|\widehat{W}|^{2} d \xi & \leq 4 n^{2} C^{2}\left(1+T_{1}\right)^{-n}(t+1)^{4 n-1} \int_{S(t)}|\xi|^{2} d \xi \\
& \leq \frac{\chi_{1}}{8}(t+1)^{7 / 2 n-2} .
\end{aligned}
$$

The last inequality follows by the choice of $T_{1}$ made at the beginning of Case

2. Combining this last bound with (4.13) and (4.14) yields

$$
\frac{d}{d t}\left[(t+1)^{4 n} \int_{\mathbf{R}^{n}}|W|^{2} d x\right] \leq \frac{\chi_{1}}{8}(t+1)^{7 / 2 n-2}+M(t+1)^{3(n-1)} .
$$


Integrating over $\left[\delta_{1}, t\right]$ gives

$$
\int_{\mathbf{R}^{n}}|W|^{2} d x \leq \frac{\chi_{1}}{8}(t+1)^{-n / 2-1}+M(t+1)^{-n-2}+\int_{\mathbf{R}^{n}}\left|W\left(x, \delta_{1}\right)\right|^{2} d x(t+1)^{-2 n} \text {. }
$$

Note that

$$
\begin{aligned}
\int_{\mathbf{R}^{n}}\left|W\left(x, \delta_{1}\right)\right|^{2} d x & \leq \int_{\mathbf{R}^{n}}|V(x, 0)|^{2} d x+\int_{\mathbf{R}^{n}}|U(x, 0)|^{2} d x \\
& \leq 2 \int\left|u\left(x, T_{1}\right)\right|^{2} d x \leq 2 \int\left|u_{0}(x)\right|^{2} d x
\end{aligned}
$$

Hence

$$
\int_{\mathbf{R}^{n}}|W|^{2} d x \leq \frac{\chi_{1}}{8}(t+1)^{-(n / 2+1)}+\text { H.O.T. }
$$

for $t$ large enough. That is for $t \geq \max \left(T_{0}, T_{2}\right)$, where

$$
T_{2}=T_{2}\left(\left|u_{0}\right|_{L^{1}},\left|u_{0}\right|_{L^{2}}, n, \alpha_{1}, \delta_{1}, \beta_{1}, C_{0}\right) .
$$

The last inequality combined with (4.11) yields

$$
|U(\cdot, t)|_{L^{2}} \geq|V(\cdot, t)|_{L^{2}}-|W(\cdot, t)|_{L^{2}} \geq(1-1 / 2) \sqrt{\chi_{1}}(t+1)^{-(n / 2+1) / 2}
$$

hence for $t \geq T_{3}=T_{1}+T_{2}$

$$
|u(\cdot, t)|_{L^{2}} \geq \frac{\sqrt{\chi_{1}}}{2}(t+1)^{-(n / 2+1) / 2} .
$$

For $t<T_{3}$ the decay of energy of $u$ yields

$$
|u(\cdot, t)|_{L^{2}}^{3} \geq\left|u\left(\cdot, T_{3}\right)\right|_{L^{2}}^{2} \geq \frac{\chi_{1}}{4}\left[\frac{1+t}{1+T_{3}}\right]^{n / 2+1}(1+t)^{-(n / 2+1)} .
$$

Thus for Case 2 let $M_{0}=\left(\chi_{1} / 4\right)\left(T_{3}+1\right)^{-(n / 2+1)}$ and the lower bound follows. In order to give the formal argument when $n=3$, the hypotheses needed are given by Case $2^{*}$ and (3.5) has to be modified adequately.

In order to make the proof rigorous for $n=3$, apply the formal proof with appropriate modifications to the subsequence $u_{\delta}$ of appropriate solutions that converges strongly in $L_{\text {loc }}^{2}\left(R_{+} \times R^{n}\right)$. For $n>3$, a similar argument should work if applied to the approximate solutions constructed by Kayikiya and Miyakawa. Let $n=3$. Let us recall that the approximation solutions $u_{\delta}$ constructed by Caffarelli, Kohn, and Nirenberg satisfy

$$
\begin{gathered}
u_{t}+\psi_{\delta} \nabla u+\nabla p=\Delta u, \\
\operatorname{div} u=0 \\
u(x, 0)=g
\end{gathered}
$$

where $\psi_{\delta}=\delta^{-4} \psi * \bar{u}$ and

$$
\begin{gathered}
\psi \in C^{\infty}, \quad \psi \geq 0, \quad \iint \psi d x d t=1, \\
\operatorname{supp} \psi \subset\left\{(x, t):|x|^{2}<t, \quad 1<t<2\right\},
\end{gathered}
$$


and let $D=\mathbf{R}^{3} \times(0, T)$,

$$
\bar{u}= \begin{cases}u(x, t) & (x, t) \in D, \\ 0 & \text { otherwise } .\end{cases}
$$

Suppose that $u_{\delta}$ is a subsequence that converges strongly to $u$ in $L_{\text {loc }}^{2}(D)$ where $u$ is a Leray-Hopf solution of Navier-Stokes equations. The steps to show that the approximate solutions satisfy

$$
\left|u_{\delta}(\cdot, t)\right|_{L^{2}} \leq M_{0}(t+1)^{-(n / 2+1)}
$$

are obtained combining the arguments of [5] with the formal proof. The lower bound for the limiting Leray-Hopf solution $u(x, t)$ follows, a.e. in $t$, taking the limit as $\delta \rightarrow 0$. The details are omitted.

\section{THE LOWER BOUNDS}

The results obtained in the previous sections are combined to establish the lower bounds for the rates of decay for solutions to the Navier-Stokes equations in two and three spatial dimensions.

Theorem 5.1. Let $u_{0} \in L^{1} \cap H\left(\mathbf{R}^{n}\right), n=2$. Then

(i) If $\hat{u}(0)=\int u(x, t) d x=\int u(x) d x \neq 0$, then there exist constants $C_{0}$ and $C_{1}$ depending only on $L^{1}$ and

$$
C_{0}(t+1)^{-n / 2} \leq|u(\cdot, t)|_{L^{2}}^{2} \leq C_{1}(t+1)^{-n / 2} .
$$

If the average $\int u(x, t) d x=\int u(x) d x=0$ then there are three cases to consider.

(ii) Let $u_{0} \in W_{1} \cap W_{2}$. Suppose that $\hat{\omega}_{0}(\xi)$ has a zero of order one at the origin; then there exist constants $C_{2}$ and $C_{3}$ such that

$$
C_{1}(t+1)^{-(n / 2+1)} \leq|u(\cdot, t)|_{L^{2}}^{2} \leq C_{2}(t+1)^{-(n / 2+1)} .
$$

The constants depend only on the $L^{2}, W_{1}$, and $W_{2}$ norms of the data.

(iii) Suppose that $u_{0} \in H^{1} \cap M^{c} \cap W_{2}$. If $\hat{u}(\xi)$ has a zero at the origin of order greater than one, then there exist constants $C_{4}, C_{5}$ such that

$$
C_{4}(t+1)^{-2} \leq|u(\cdot, t)|_{L^{2}}^{2} \leq C_{5}(t+1)^{-2},
$$

where $C_{4}$ depends only on the $L^{1}$ and $L^{2}$ norms of $u_{0}$ and $C_{5}$ depends on the $L^{1}, L^{2}, H^{1}, W_{2}$ norms of the data and

Proof.

$$
\alpha=\int_{\mathbf{R}^{2}}\left|u_{1}^{0}\right|^{2}-\left|u_{2}^{0}\right|^{2} d x \quad \text { or } \beta=\int_{\mathbf{R}^{2}} u_{1}^{0} u_{2}^{0} d x .
$$

Upper bound. Case (i). See [5]. Cases (ii), (iii) follow from Theorems 2.1, $3.2,3.3$ and the decay rates for the difference $\omega$ between the solution to the heat equation and the solution to the Navier-Stokes equation obtained by Wiegner [6]. 
Lower bound. Case (i). The proof can be found in [5, Theorem (4.2)]. There are a few simple modifications.

1. The more restrictive hypothesis of Theorem (4.2) [5], $|\hat{u}(\xi)| \neq 0$ for $|\xi| \leq \delta$, can be deduced from $\hat{u}(0) \neq 0$, since $u_{0} \in L^{1}$ implies $\hat{u} \in C^{1}$.

2. The necessary upper bounds for $\omega$ when $n=2$ can be found in [6].

Case (ii). Follows from Theorems 2.1, 3.1, and 4.1.

Case (iii). Follows from Theorems 2.1, 3.2, and 4.1.

Theorem 5.2. Let $u_{0} \in L^{1} \cap H\left(\mathbf{R}^{n}\right), n=3$. Let $u(x, t)$ be a suitable LerayHopf solution in the sense of Caffarelli, Kohn, and Nirenberg and the lower bound holds for almost all $t$. Then (i) and (ii) of Theorem 5.1 hold, where the upper bound is for all $t$ and the lower bound is for a.e. $t$.

(iii) If there exists $t_{0}$ such that $u \in M_{t_{0}}^{c}$ and also $u_{0} \in W_{2}$, then

$$
C_{6}(t+1)^{-5 / 2} \leq|u(\cdot, t)|_{L^{2}}^{2} \leq C_{7}(t+1)^{-5 / 2},
$$

where $C_{7}$ depends only on the $L^{1}$ and $L^{2}$ norms of the data and $C_{6}$ depends on the $L^{1}, L^{2}, W_{2}$ norms of the data and $\alpha_{j}^{i}=\alpha_{j}^{i}\left(t_{0}, 3\right)$ and $\beta_{j}^{i}=\beta_{j}^{i}\left(t_{0}, 3\right)$, where $\alpha_{j}^{i}$ and $\beta_{j}^{i}$ were defined in Theorem 3.

Proof.

Upper bound. See Theorem 5.1, apply proof to approximation solutions, and pass to the limit.

Lower bound. Cases (i), (ii). Same steps as in Theorem 5.1 applied to special subsequence of approximate solutions, which is supposed to exist by hypothesis and pass to limit. Bound will be valid for a.e. $t$.

Case (iii). Follows from Theorems 2.1, 3.3, and 4.1 applied to special sequence of approximate solutions existing by hypothesis and pass to the limit. The bound will hold for almost all $t$.

We expect that Theorems 5.1 and 5.2 can be extended to $n$ dimensions for all $n \geq 4$ using the approximate solutions constructed by Kayikiya and Miyakawa. The hypothesis on the data $u_{0} \in M^{c}$ for $n=2$ is optimal as the following example shows. In three dimensions we expect that the condition $u \in M_{t_{0}}^{c}\left(\mathbf{R}^{3}\right)$ is necessary but we have not found an example where the lower bound fails if $u \in M_{t_{0}}\left(\mathbf{R}^{3}\right)$. The example in two dimensions we will present was suggested by A. Majda.

Example 5.3. Exponentially decaying vorticity in 2 spatial dimensions with data $u_{0} \in M$.

Let $u(x, t)$ be a solution to the $2 \mathrm{D}$ Navier-Stokes equation with radial vorticity. Suppose also that $u_{0} \in M \cap L^{1} \cap L^{2}$ is such that curl $u_{0}=\omega_{0}$ satisfies

(i) $\omega_{0} \in L^{1}$,

(ii) $\nabla u_{0} \in L^{1}$,

(iii) $\hat{\omega}(\xi)=0$ for $|\xi| \leq \delta$, some $\delta>0$. 
Then

$$
u(x, t)=\left(\begin{array}{c}
-x_{2} / r^{2} \\
x_{1} / r^{2}
\end{array}\right) \int_{0}^{r} s \omega(s) d s, \quad r^{2}=x_{1}^{2}+x_{2}^{2},
$$

where $\omega$ is the vorticity. Here $u_{0}(x)=u(x, 0) \in M\left(\mathbf{R}^{2}\right)$, since

and

$$
\int_{\mathbf{R}^{2}} u_{1}^{2}(x, 0) d x=\int_{\mathbf{R}^{2}} u_{2}^{2}(x, 0) d x
$$

The vorticity equation is reduced to

$$
\int_{\mathbf{R}^{2}} u_{1}^{0}(x, 0) u_{2}^{0}(x, 0) d x=0 .
$$

$$
\begin{gathered}
\omega_{t}=\Delta \omega, \\
\omega(|x|, 0)=\omega(x, 0)=\omega_{0}(r) .
\end{gathered}
$$

The term $u \cdot \nabla \omega$ is zero, since the vorticity is radial. By (iii) follows

$$
\begin{aligned}
\int_{\mathbf{R}^{2}}\left|\omega(x, t)^{2}\right| d x & =\int_{\mathbf{R}^{2}}|\hat{\omega}(\xi, t)|^{2} d \xi=\int_{\mathbf{R}^{2}}\left|\hat{\omega}_{0}(\xi)\right|^{2} e^{-|\xi|^{2} t} d \xi \\
& \leq e^{-\delta^{2} t} \int_{\mathbf{R}^{2}}\left|\hat{\omega}_{0}(\xi)\right|^{2} d \xi
\end{aligned}
$$

Hence $|\omega|_{L^{2}}^{2} \leq k e^{-|\delta|^{2} t}$. We only give an outline for the estimate

$$
\int_{\mathbf{R}^{2}}|u|^{2} d x \leq C_{0} \exp \left(-C_{1} t\right)
$$

We use the explicit form of $u$ and the decay of $|\omega|_{L^{2}}$. This estimate will follow from the explicit form of $u, v$ and the decay of $|\omega|_{L^{2}}$.

By the Jensen inequality and (5.1) it follows that

$$
\begin{aligned}
|u|^{2}+|v|^{2} & \leq \frac{1}{r^{2}}\left(\int_{0}^{r} s \omega d s\right) \leq \int_{0}^{r} s^{2} \omega^{2} \frac{d s}{r} \leq \frac{1}{2 \pi} \int_{0}^{2 \pi} \int_{0}^{r} s \omega^{2} d s \\
& =\frac{1}{2 \pi} \int_{\mathbf{R}^{2}}|\omega|^{2} d x \leq C_{0} e^{-\delta^{2} t}
\end{aligned}
$$

hence

$$
|u|_{\infty}+|v|_{\infty} \leq C \exp \left(-\delta^{2} t / 2\right)
$$

The $L^{2}$ decay of $u$ will follow from a time dependent $L^{1}$ estimate, more precisely from

$$
|u(\cdot, t)|_{L^{1}} \leq C t
$$

where $C$ depends on $L^{1}, L^{2}$ norms of $u$ and $\omega$ and also on the $L^{1}$ norm of $\nabla \omega$.

\section{APPENDIX}

Proposition A.1. Let $u_{0} \in H\left(\mathbf{R}^{2}\right) \cap W_{2}$. Then if $u$ is a solution of Navier-Stokes with data $u_{0}$

$$
\left|\nabla_{\xi} a_{i j}(\xi, t)\right| \leq C(t),
$$

where $a_{i j}={\widehat{u_{i} u_{j}}}_{\text {and }} C(t)$ depends on $\left|u_{0}\right|_{L^{2}}$. 
Proof.

$$
\left|\partial_{\xi_{k}} a_{i j}(\xi, t)\right| \leq \int_{\mathbf{R}^{2}}\left|x_{k}\right|\left|u_{i} u_{j}\right| d x \leq \int_{\mathbf{R}^{2}}\left|x_{k}\right||u|^{2} d x .
$$

Hence we need to bound $\int_{\mathbf{R}^{2}}|x||u|^{2} d x$ in terms of the initial data. Multiply the Navier-Stokes equation by $\left|x_{k}\right| u_{j}$ and integrate in space. We do it componentwise and sum

(A.2)

$$
\begin{aligned}
\begin{aligned}
\frac{d}{d t} \int_{\mathbf{R}^{2}}\left|x_{k}\right| u_{j} u_{j} d x & =-\int_{\mathbf{R}^{2}}\left|x_{k}\right| u_{j} \sum_{i} u_{i} \partial_{i} u_{j} d x \\
& -\int_{\mathbf{R}^{2}}\left|x_{k}\right| u_{j} \partial_{j} p d x+\sum_{s} \int_{\mathbf{R}^{2}}\left|x_{k}\right| u_{j} \partial_{s s} u_{j} d x \\
& =\mathrm{I}+\mathrm{II}+\mathrm{III} ; \\
\mathrm{I} & =\frac{1}{2} \sum_{i} \int_{\mathbf{R}^{2}} \operatorname{sign} x_{k} u_{i} u_{j}^{2} d x \leq \frac{1}{2} \int_{\mathbf{R}^{2}}|u|^{2}+\frac{1}{2} \int_{\mathbf{R}^{2}}|u|^{4} \\
& \leq \frac{1}{2} \int_{\mathbf{R}^{2}}|u|^{2}+\frac{1}{2} \int_{\mathbf{R}^{2}}|\nabla u|^{2},
\end{aligned}
\end{aligned}
$$$$
\text { where } \int|u|^{4} \text { follows by Ladyzenkaya [3, Lemma 7]. }
$$

For the second term it is convenient to bound the sum of all the terms and use that $u$ is divergent free:

$$
\begin{aligned}
\sum_{j} \mathrm{II} & \leq \int_{\mathbf{R}^{2}}\left|\operatorname{sign}\left(x_{k}\right)\right|\left|u_{j}\right||p| d x \\
& \leq \int_{\mathbf{R}^{2}}|u|^{2}+\int_{\mathbf{R}^{2}}|p|^{2} \leq \int_{\mathbf{R}^{2}}|u|^{2}+4 \int_{\mathbf{R}^{2}}|u|^{4} \leq \int_{\mathbf{R}^{2}}|u|^{2}+\int_{\mathbf{R}^{2}}|\nabla u|^{2} .
\end{aligned}
$$

The bound on $p$ above follows by recalling that the pressure satisfies an elliptic equation, which is obtained by taking the divergence of the Navier-Stokes equation,

$$
\Delta p=-\sum_{i, j} \frac{\partial^{2}}{\partial x_{i} \partial x_{j}} u_{i} u_{j}
$$

hence

$$
\hat{p}=-\sum_{i, j} \frac{\xi_{i} \xi_{j}}{|\xi|^{2}}{\widehat{u_{i} u_{j}}}
$$

and

$$
\begin{aligned}
\int_{\mathbf{R}^{2}}|p|^{2} d x & =\int_{\mathbf{R}^{2}}|\hat{p}|^{2} d \xi \leq \int_{\mathbf{R}^{2}} \sum_{i, j} \frac{\xi_{i} \xi_{j}}{|\xi|^{2}}{\widehat{u_{i} u_{j}}}_{j} \xi \\
& \leq 4 \int_{\mathbf{R}^{2}}|u|^{4} d x \leq 8 \int_{\mathbf{R}^{2}}|\nabla u|^{2} d x
\end{aligned}
$$


Finally III is estimated as follows:

$$
\begin{aligned}
\text { III } & =\sum_{s} \int_{\mathbf{R}^{2}} \operatorname{sign}\left(x_{k}\right) u_{j} \partial_{s} u_{j} d x-\int_{\mathbf{R}^{2}}\left|x_{k}\right|\left|\nabla u_{j}\right|^{2} d x \\
& \leq 3 \int_{\mathbf{R}^{2}}|u|^{2} d x+\int_{\mathbf{R}^{2}}|\nabla u|^{2} d x .
\end{aligned}
$$

Hence integrating over $[0, t]$ (A.2) and combining (A.1), (A.2), (A.3), (A.4), and (A.6)

$$
\begin{aligned}
\left|\partial_{\xi_{k}} a_{i j}(\xi, t)\right| & \leq \int_{\mathbf{R}^{2}}\left|x_{k}\right||u|^{2} d x \\
& \leq C\left(\int_{0}^{t} \int_{\mathbf{R}^{2}}|u|^{2} d x d s+\int_{0}^{t} \int_{\mathbf{R}^{2}}|\nabla u|^{2} d x d s\right)+\int_{\mathbf{R}^{2}}\left|x_{k}\right|\left|u_{0}\right|^{2} \\
& \leq C(t+1) \int_{\mathbf{R}^{2}}\left|u_{0}\right|^{2} d x+\int_{\mathbf{R}^{2}}\left|x_{k}\right|\left|u_{0}\right|^{2} d x,
\end{aligned}
$$

where $C \leq 40$.

Lemma A.2. Let $u_{0}=\left(u_{0}^{1}, u_{0}^{2}\right) \in M^{c} \cap H^{1}\left(\mathbf{R}^{2}\right)$. Let $\alpha=\int_{\mathbf{R}^{2}}\left|u_{0}^{1}\right|^{2}-\left|u_{0}^{2}\right|^{2} d x \neq$ 0 . There then exists $t_{0}$ such that if $u(x, t)$ is a solution to the Navier-Stokes equation with data $u_{0}$

$$
\left.\left|\int_{0}^{T} \int_{\mathbf{R}^{2}}\right| u_{1}\right|^{2}-\left|u_{2}\right|^{2} d x d t \mid \geq(\alpha / 2) T
$$

for all $T \leq t_{0}, t_{0}$ depending only on the $H^{1}$ norm of $u_{0}$.

Proof. W.l.o.g. suppose that $\alpha>0$. If not take

$$
\alpha=\int_{\mathbf{R}^{2}}\left|u_{0}^{2}\right|^{2}-\left|u_{0}^{1}\right|^{2} d x
$$

Let

$$
F(t)=\int_{\mathbf{R}^{2}}\left|u_{1}\right|^{2}-\left|u_{2}\right|^{2} d x .
$$

The following estimate is needed.

$$
\left|\frac{d}{d t} F(t)\right| \leq C \int_{\mathbf{R}^{2}}|\nabla u|^{2} d x .
$$

The last estimate follows from multiplying the equation for the first component of Navier-Stokes by $u_{1}$, the second by $u_{2}$, and integrating in space. Hence

$$
\begin{aligned}
&\left|\frac{d}{d t} F(t)\right| \leq \mid \int_{\mathbf{R}^{2}} u_{1} \sum_{i} u_{i} \partial_{i} u_{1}-u_{1} \partial_{1} p+u_{1} \Delta u_{1} d x \\
&-\int_{\mathbf{R}^{2}} u_{2} \sum_{i} u_{i} \partial_{i} u_{2}-u_{2} \partial_{2} p+u_{2} \Delta u_{2} d x \mid .
\end{aligned}
$$

Thus from (A.5)

$$
\left|\frac{d}{d t} F(t)\right| \leq 2 n \int_{\mathbf{R}^{2}}|\nabla u|^{2} d x+\int_{\mathbf{R}^{2}}|u|^{4} d x .
$$


By Lemma (7) in [3] it follows that if $|u(x, t)| \rightarrow 0$ as $|x| \rightarrow \infty$,

$$
\int_{\mathbf{R}^{2}}|u|^{4} d x \leq 2 \int_{\mathbf{R}^{2}}|\nabla u|^{2} d x \int_{\mathbf{R}^{2}}|u|^{2} d x \leq C \int_{\mathbf{R}^{2}}|\nabla u|^{2} d x
$$

with $C=4 n C_{0}, C_{0}=\left|u_{0}\right|_{L^{2}}^{2}$. Recall that solutions to the Navier-Stokes equations in two spatial dimensions satisfy

$$
\int_{\mathbf{R}^{2}}|\nabla u|^{2} d x \leq \int_{\mathbf{R}^{2}}\left|\nabla u_{0}\right|^{2} d x
$$

The last inequality follows from the special relation between the partial differentials of the components of the gradient. This relation is a consequence of the solution being divergence free. By the mean value theorem it follows that

$$
|F(t)-F(0)| \leq\left|F^{\prime}(\bar{s})\right| t \leq C t \int_{\mathbf{R}^{2}}|\nabla u|^{2}(\bar{s}) d x \leq C t \int_{\mathbf{R}^{2}}\left|\nabla u_{0}\right|^{2} d x .
$$

Thus $F(t) \geq F(0)-C_{1} t$, where $C_{1}=C \int_{\mathbf{R}^{2}}\left|\nabla u_{0}\right|^{2} d x$. Integrating over $[0, T]$ yields

$$
\int_{0}^{T} F(t) d t \geq F(0) T-C_{1} T^{2} / 2
$$

and for any $T \leq F(0) / 2 C_{1}$ it follows that

$$
\int_{0}^{T} F(t) d t \geq F(0) T / 2
$$

Let $t_{0}=F(0) / 2 C_{1}$, and the lemma follows.

Lemma A.3. Let $u_{0}=\left(u_{0}^{1}, u_{0}^{2}\right) \in N^{c} \cap H^{1}$. Let $\alpha=\int_{\mathbf{R}^{2}} u_{0} u_{1} d x \neq 0$. Then there exists $t_{0}>0$ such that

$$
\left|\int_{0}^{T} \int_{\mathbf{R}^{2}} u_{0} u_{1} d x\right| \geq \alpha / 2 T
$$

for all $T \leq t_{0}, t_{0}$ depending only on the $H^{1}$ norms of $u_{0}$.

Proof. W.l.o.g. suppose $\alpha>0$. Following the lines of the proof for Lemma A. 2 we only need to show that

$$
\left|\frac{d}{d t} G(t)\right| \leq C \int_{\mathbf{R}^{2}}\left|\nabla u_{0}\right|^{2} d x,
$$

where

$$
G(t)=\int_{\mathbf{R}^{2}} u_{0} u_{1} d x
$$


Note that

$$
\begin{aligned}
\left|\frac{d}{d t} G(t)\right| \leq\left|\int_{\mathbf{R}^{2}} u_{1} u_{2 t}+u_{1 t} u_{2} d x\right| \\
=\mid-\int_{\mathbf{R}^{2}} u_{1} \sum_{i} u_{i} \partial_{i} u_{2} d x-\int_{\mathbf{R}^{2}} u_{1} \partial_{2} p d x+\int_{\mathbf{R}^{2}} u_{1} \Delta u_{2} d x \\
\quad-\int_{\mathbf{R}^{2}} u_{2} \sum_{i} u_{i} \partial_{i} u_{1} d x-\int_{\mathbf{R}^{2}} u_{2} \partial_{1} p d x+\int_{\mathbf{R}^{2}} u_{2} \Delta u_{1} d x \mid \\
\leq C\left(\int_{\mathbf{R}^{2}}|u|^{4} d x+\int_{\mathbf{R}^{2}}|\Delta u|^{2} d x+\int_{\mathbf{R}^{2}}|p|^{4} d x\right)
\end{aligned}
$$

and from the observation in the previous lemma it follows that last term is bounded by the $L^{2}$ norm of the gradient of $u$ and hence

$$
\left|\frac{d}{d t} G(t)\right| \leq C \int_{\mathbf{R}^{2}}\left|\nabla u_{0}\right|^{2} d x .
$$

And the proof now is the same as for Lemma A.2.

Note A.4. We recall that in Lemma (8.2) [1], Caffarelli, Kohn, and Nirenberg showed that for a suitable Leray-Hopf solution for almost all $t$

$$
\int_{\mathbf{R}^{3}}|u(x, t)|^{2}|x| d x \leq A(t)
$$

with $A(t)$ depending only on the $L^{2}$ and $W_{2}$ norms of the data. Let

$$
A=\left\{t:\left|\frac{\partial}{\partial \xi}{\widehat{u_{i}}}_{j}(\xi, t)\right| \leq A(t)\right\},
$$

where $u=\left(u_{1}, u_{2}, u_{3}\right)$ is a suitable Leray-Hopf solution to Navier-Stokes. By Lemma 8.2 [1] such a set is nonempty. Moreover, $A$ can be chosen so that $m\left(A^{c}\right)=0$. With the notation $a_{i j}={\widehat{u_{i} u_{j}}}_{1} a_{i j}^{0}(t)={\widehat{u_{i} u_{j}}}(0, t)$ iff $t \in A$

$$
a_{i j}(\xi, t)=a_{i j}^{0}(t)+\xi \cdot \nabla_{\xi} a_{i j}(\bar{\xi}, t) \text {. }
$$

\section{ACKNOWLEDGMENTS}

The suggestion to look at the lower bounds of rates of solutions decay the Navier-Stokes equation was made by Ron DiPerna. I would like to thank A. Majda for his helpful comments. I would also like to thank the referee for his careful reading of the work and his many helpful suggestions.

\section{REFERENCES}

1. L. Caffarelli, R. Kohn, and L. Nirenberg, Partial regularity of suitable weak solutions of the Navier-Stokes equations, Comm. Pure Appl. Math. 35 (1982), 771-831.

2. R. Kayikiya and T. Miyakawa, On the $L^{2}$ decay of weak solutions of the Navier-Stokes equations in $\mathbf{R}^{n}$, Math. Z. 192 (1986), 135-148.

3. O. A. Ladyzenkaya, The mathematical theory of viscous incompressible flows, 2nd ed., Gordon and Breach, New York, 1969. 
4. M. E. Schonbek, $L^{2}$ decay for weak solutions of the Navier-Stokes equations, Arch. Rational Mech. Anal. 88 (1985), 209-222.

5. __ Large time behavior of solutions to the Navier-Stokes equations, Comm. Partial Differential Equations 11 (1986), 753-763.

6. M. Wiegner, Decay results for weak solutions of the Navier-Stokes equations in $\mathbf{R}^{n}, \mathbf{J}$. London Math. Soc. (2) 35 (1987), 303-313.

Department of Mathematics, University of California, Santa Cruz, California 95064 